\title{
The effect of microhydration on ionization energies of thymine
}

\author{
Kirill Khistyev $^{a}$, Ksenia B. Bravaya ${ }^{a}$, Eugene Kamarchik ${ }^{a, b}$, \\ Oleg Kostko ${ }^{c}$, Musahid Ahmed ${ }^{c}$, and Anna I. Krylov ${ }^{a}$ \\ ${ }^{a}$ Department of Chemistry, University of Southern California, \\ Los Angeles, CA 90089-0482, USA \\ ${ }^{b}$ Department of Chemistry, C.L. Emerson Center for Scientific Computation, Emory University, \\ Atlanta, Georgia 30322, USA \\ ${ }^{c}$ Chemical Sciences Division, Lawrence Berkeley National Laboratory, Berkeley, CA 94720, USA
}

A combined theoretical and experimental study of the effect of microhydration on ionization energies (IEs) of thymine is presented. The experimental IEs are derived from photoionization efficiency curves recorded using tunable synchrotron VUV radiation. The onsets of the PIE curves are $8.85 \pm 0.05,8.60 \pm 0.05,8.55 \pm 0.05$, and $8.40 \pm 0.05 \mathrm{eV}$ for thymine, thymine mono-, di-, and tri-hydrates, respectively. The computed (EOM-IP-CCSD/cc-pVTZ) AIEs are 8.90, 8.51, 8.52, and $8.35 \mathrm{eV}$ for thymine and the lowest isomers of thymine mono-, di-, and tri-hydrates. Due to large structural relaxation, the Franck-Condon factors for the $0 \leftarrow 0$ transitions are very small shifting the apparent PIE onsets to higher energies. Microsolvation strongly affects IEs of thymine - addition of each water molecule reduces the first vertical IE by $0.10-0.15 \mathrm{eV}$. The adiabatic IE decreases even more (up to $0.4 \mathrm{eV}$ ). The magnitude of the effect varies for different ionized states and for different isomers. For the ionized states that are localized on thymine the dominant contribution to the IE reduction is the electrostatic interaction between the delocalized positive charge on thymine and the dipole moment of the water molecule.

\section{INTRODUCTION}

Ionization of nucleic acid bases (NABs) is involved in DNA radiation and photo-damage and may eventually lead to dangerous mutations with a risk for cancer and neurodegenerative diseases. Due to their relatively low ionization energies (IEs), individual nucleobases are the most likely components of DNA to be oxidized. Their IEs, however, are affected by 
coupling to DNA's sugar-phosphate backbone, hydrogen-bonding and $\pi$-stacking between neighboring bases, as well as interactions with solvating water molecules and counter ions. Quantifying the exact nature of these effects has proven difficult, and even the values of IEs of solvated DNA are still controversial. For example, a recent computational study[1] that attempted to compute the IE of guanine in solvated DNA by a QM/MM approach reported a large increase $(4 \mathrm{eV})$ of guanine's IE, contrary to the conclusions drawn from experimental studies[2].

As part of our effort to understand the role played by different interactions of NABs with the environment[3-8], we recently characterized the effect of hydrogen-bonding and $\pi$ stacking on the ionized states of the gas-phase dimers of AA, TT, AT, and CC in a combined experimental and theoretical study $[7,8]$. We found that stacking and hydrogen-bonding can reduce the IEs by $0.4-1 \mathrm{eV}$ via two distinct mechanisms: hole delocalization and electrostatic charge-dipole interactions. We also analyzed ionization-induced structural changes in isolated nucleic acid bases[9] and in uracil dimers[4,5]. Consistent with delocalized character of the highest occupied molecular orbital (HOMO), structural changes involve several CC and CN bonds, the largest change being for the double CC bond. In bare NABs the relaxation energy is $0.2-0.4 \mathrm{eV}$, whereas in the dimers the difference between adiabatic and vertical IEs is larger. To quantify the effect of structural relaxation on photoionization efficiency (PIE) curves, we computed Franck-Condon factors (FCFs) for the lowest-energy tautomers of $\mathrm{NABs}[9]$. In all cases, we observed that the $0 \longleftarrow 0$ transitions have non-negligible FCFs and that the onsets of the PIE curves indeed represent AIEs.

Microsolvation has been found to decrease the IEs of nucleobases by about $0.1 \mathrm{eV}$ per water molecule[6, 10]. The absolute values of IEs reported in VUV[6] and electron impact[10] studies are slightly different. An excellent agreement of the IE of isolated thymine determined from the PIE curve $(8.90 \pm 0.05 \mathrm{eV})[6]$ with the value derived from MATI spectra $(8.9178 \pm 0.0010 \mathrm{eV})[11]$ validates the accuracy of the synchrotron VUV measurements[6].

The computational studies[12, 13] performed with B3LYP predicted similar magnitude shifts and pointed out substantial geometric relaxation in hydrated species leading to even larger changes in AIEs. These studies were motivated by differences between the results obtained by the two experimental approaches, i.e., using electron impact ionization and VUV photoionization $[6,10]$. A number of tautomers were calculated to interpret the early electron impact results, while attempts were made to fit the appearance energy measurements to 
various vertical and adiabatic values.

In other nucleobases, microhydration leads to similar effects[6], although the magnitude of the IE drop varies. For example, the changes in AIE in adenine-water clusters $[6,14]$ are smaller than those for thymine.

The effect of microsolvation on electronically excited states and photoinduced dynamics in nuclear bases and other model chromophores has been investigated theoretically and experimentally[15-22]. In addition to perturbations to the electronic spectrum and differential stabilization of excited states, microsolvation can open up new relaxation channels including, among others, hydrogen/proton and electron transfer, charge-transfer-to-solvent states, and zwitter-ion formation.

The theoretical treatment of ionized species is challenging owing to their open-shell character and electronic near-degeneracies[23, 24]. Wave-function approaches using doublet references often suffer from spin-contamination and symmetry-breaking, which result in hole over-localization[23]. DFT methods are affected by self-interaction error leading to charge over-delocalization. Owing to these defects, computational studies often observe artifacts of electronic structure methodology rather than real physical properties of these systems. We employ the EOM-IP method that is free from the above problems and is the method of choice for these systems. EOM-IP describes open-shell target states as ionized states derived from well behaved closed-shell neutral reference wave functions (see Section II A). We also use DFT with a range-separated functional ( $\omega$ B97X-D) that greatly reduces self-interaction errors $[25,26]$.

The appearance energies of microhydrated thymine ions $\left.\left[\mathrm{T}\left(\mathrm{H}_{2} \mathrm{O}\right)_{n}, \mathrm{n}=1-3\right)\right]$ have been reported previously by our group[6]. In the present work we have performed the measurements with a broader energy range and report improved error bars. Furthermore, derivation of the PIE curves as reported by us for NABs and their dimers, allows for qualitative interpretation of the VIEs for thymine and hydrated thymine as a maxima at obtained curves. In the earlier experimental work[6], the beam contained mixed adenine-thymine and water clusters, and in the new experiments reported here thymine alone was microhydrated with water.

Previous experimental and theoretical studies on the microhydration of thymine do not provide a detailed physical picture of the ionization processes. Hence, the focus of this work 
is on quantifying the effect of microsolvation on IEs and on understanding its origin. We consider several isomers of the microhydrates shown in Fig. 1 (all structures correspond to the lowest-energy tautomer of thymine). In order to unambiguously compare with the experimental measurements, we also perform modeling of the FCFs for the lowest electronic state of the cations. Accurate FCF calculations for hydrogen-bonded systems of such complexity are rare and provide important benchmarks as well as insight into the spectroscopy of biologically relevant species.

\section{EXPERIMENTAL AND COMPUTATIONAL TECHNIQUES}

\section{A. Electronic structure calculations}

Open-shell doublet wave functions can be formally derived from a closed-shell systems by addition or subtraction of an electron. As such, they can be described accurately and efficiently by the ionization potential (IP) and electron affinity (EA) variants of equation-ofmotion coupled-cluster (EOM-CC) methods [27-30]. EOM-IP, which relies on the N-electron closed-shell reference, is free from the symmetry breaking and spin-contamination problems that are ubiquitous in open-shell calculations, and is capable of describing charge localization patterns in ionized clusters[4, 7, 23, 31]. EOM-IP simultaneously includes dynamical and non-dynamical correlation, describes multiple electronic states in one calculation, and treats states with different number of electrons on the same footing. Using the EOM-CC family of methods, electronically excited, ionized, or attached states of the thymine-water clusters can be computed starting from the same closed-shell CCSD (coupled-cluster with singles and doubles) reference wave function of the neutral[24, 32].

The target open-shell wave functions are generated by a Koopmans-like excitation operator $R$ acting on the reference $\mathrm{CC}$ wave function:

$$
\Psi^{E O M-I P}(N-1)=\hat{R} e^{\hat{T}} \Phi_{0}(N)
$$

where $\Phi_{0}(N)$ is the reference determinant of the $N$-electron neutral system, $T$ is the coupledcluster excitation operator including single and double substitutions, and $R$ consists of $1 h$ and $2 h 1 p$ (1-hole and 2-hole-1-particle) operators generating $(N-1)$-electron determinants from the $N$-electron reference. Amplitudes $T$ are found by solving CCSD equations for the 
ground-state wave function of the neutral, while amplitudes $R$ are obtained by subsequent diagonalization of the similarity transformed Hamiltonian, $\bar{H}=e^{-T} H e^{T}$.

EOM-IP-CCSD yields accurate energy splittings and smooth potential energy surfaces along charge transfer coordinates[23]. This method has been successfully applied to describe electronic structure of ionized benzene dimers[31, 33], water clusters[34-36] and dimers of nucleobases $[3-5,7,8]$.

Typical errors in excitation energies associated with "single excitation processes" for EOM-CC with single and double substitutions are in the $0.10 .3 \mathrm{eV}$ range $[37,38]$. However, energy differences between different ionized states are described much better (errors below $0.05 \mathrm{eV}$ ) [23]. In our recent joint experimental and theoretical study of the four gas-phase nucleobases, the errors in computed adiabatic IEs relative to the experimental values were found to be below $0.1 \mathrm{eV}[9]$.

We also employed DFT with the long-range and dispersion-corrected $\omega$ B97X-D functional [26] (for geometry optimization and frequency calculations). Long-range Hartree-Fock exchange included in $\omega$ B97X-D mitigates the effect of self-interaction error yielding accurate structures and frequencies[7, 9].

There are several tautomers of thymine; however, the energy gap between the canonical form and next most stable tautomer is more than $10 \mathrm{kcal} / \mathrm{mol}[39]$ in the gas phase. Since we used thermal vaporization to generate thymine in the gas phase, there is not enough energy to populate higher-lying tautomers. Thus, the canonical form should be predominantly present in a molecular beam.

We considered the three most stable monohydrated thymine isomers (T1-T3, see Fig. 1) and the two thymine- $\left(\mathrm{H}_{2} \mathrm{O}\right)_{2}$ structures (T11, T12) obtained by Hobza et al. using the molecular dynamics/quenching technique (MD/Q)[39]. The fourth most stable monohydrate structure (T4) shown in Fig. 1 is $4.4 \mathrm{kcal} / \mathrm{mol}$ higher in energy than the most stable T1. Therefore we excluded T4 from further consideration. Thymine trihydrate structures (T111 and T112) were obtained by addition of a water molecule to dihydrate structures. All ground-state geometries were optimized using the RI-MP2/cc-pVTZ [40, 41] and $\omega B 97 X-D / c c-p V T Z$ methods, which yielded similar results. For example, the differences in all bond lengths for the thymine molecule in $\mathrm{T} 1$ is less than $0.01 \AA$; the hydrogen bond between the N-H group of thymine and the oxygen of water is $0.028 \AA$ shorter and the 
hydrogen bond between $\mathrm{C}=\mathrm{O}$ of thymine and the hydrogen of water is $0.026 \AA$ shorter for RI-MP2 (1.871 and $1.903 \AA$ respectively) than for $\omega$ B97X-D (1.899 and $1.929 \AA$, respectively). Figs. 2 and 3 show the RI-MP2 ground states geometries. The respective Cartesian geometries as well as $\omega$ B97X-D structures are given in Supplementary Materials.

The equilibrium structures of the cations were computed with $\omega$ B97X-D/cc-pVTZ (see Figs.4 and 5). This functional, when used with 6-311++G(3df,3pd) basis set, was shown to describe geometries and binding energies of the weakly bound complexes with mean absolute errors of $0.064 \AA$ and $0.22 \mathrm{kcal} / \mathrm{mol}$, respectively[26]. For the ionized thymine monohydrates, this functional (with the cc-pVTZ basis) yields structures that are similar to those obtained with EOM-IP-CCSD/6-311+G(d,p) [42], i.e., the mean absolute errors are $0.01 \AA$ for the thymine moiety and $0.11 \AA$ for thymine-water hydrogen bond.

All IEs were calculated using EOM-IP-CCSD/cc-pVTZ at the equilibrium geometries described above. The cc-pVTZ basis set provides a good balance between accuracy and computational efficiency. The first IE of thymine computed using EOM-IP-CCSD with the extended cc-pVTZ basis [augmented by diffuse s and p functions from 6-311++G(d,p) as was done in Ref. [43]] is $9.20 \mathrm{eV}$, which is $0.07 \mathrm{eV}$ higher than the cc-pVTZ value. Zero point energy (ZPE) correction lowers AIEs of thymine and thymine monohydrates (T1 and T2) by $0.08 \mathrm{eV}$. Thus, due to error cancellation, non ZPE-corrected AIEs computed with cc-pVTZ are very close to the ZPE-corrected AIEs obtained with the extended cc-pVTZ basis set.

The charge distribution analysis were performed using Natural Bond Orbital Package (NBO, v. 5.0) [44]. All calculations were conducted using the Q-CHEM electronic structure package[45]. Molecular structures, frequencies, and relevant total energies are given in Supplementary Materials.

\section{B. Calculation of the Franck-Condon factors and PIE curves}

Unambiguous comparison with the experimental PIE curves requires calculation of FCFs. While in molecular systems (i.e., ionized NABs), FCFs can be reliably computed using the 
double-harmonic approximation with Duschinsky rotations[46], as was done in Ref. [9], the calculations in clusters are more challenging due to large structural relaxation of soft (and anharmonic) inter-fragment degrees of freedom. To correctly describe these effects, we combine double-harmonic treatment of the thymine moiety and water with a one-dimensional quantum treatment of the inter-fragment coordinate assuming that the water-thymine and intramolecular thymine vibrations are uncoupled and that the respective FCFs are multiplicative.

Using the ezSpectrum program [47], we first compute FCFs for the thymine moiety using double-harmonic approximation with Duschinsky rotations[46] at the $\mathrm{C}_{s}$ geometry using normal modes and frequencies for the non-planar structures with one hydrogen of water out of the plane. The water molecule itself was excluded from this calculation and only the geometry of the thymine moiety (from the monohydrate) was used. Duschinsky rotations are important because the normal-mode overlap matrix for the neutral and the ionized states is significantly non-diagonal. In these calculations, we used harmonic frequencies and structures computed by $\omega$ B97X-D/cc-pVTZ for both the neutral and the 1st ionized states of the monohydrates.

The effect of water-thymine degrees of freedom on FCFs is described by a one-dimensional model, which takes into account anharmonicity and large structural relaxation. This treatment is similar to the intrinsic reaction coordinate connecting the initial and final state. Water-thymine motion is defined by three coordinates: $r$, the distance between the water oxygen and the nearest hydrogen in thymine, $\theta$, the angle formed by the NH bond in thymine and the oxygen in water, and $\varphi$, the rotation of the water center-of-mass relative to the axis defined by $r$ (Fig.6). In the neutral thymine monohydrate (T1), these coordinates obtain the values $r=1.923 \AA, \theta=36.7^{\circ}$, and $\varphi=40.6^{\circ}$, while in the cation they equal $r=1.668$ $\AA, \theta=1.6^{\circ}$, and $\varphi=2.2^{\circ}$. Along the simplest path, each coordinate is described by a linear equation connecting the values in the neutral and in the cation. This path is used to evaluate two potential surfaces, V(r), one with the thymine held at its equilibrium neutral geometry and the second with thymine held at its equilibrium cation geometry. The effective one-dimensional Hamiltonian describing this motion is:

$$
\hat{H}=-\frac{1}{2 m r} \frac{\partial}{\partial r}-\frac{1}{2 m} \frac{\partial^{2}}{\partial r^{2}}-\frac{1}{2 m r^{2}} \frac{\partial^{2}}{\partial \theta^{2}}-\frac{1}{2 I} \frac{\partial^{2}}{\partial \varphi^{2}}+\hat{V}(r),
$$

where $m$ is the mass of water and $I$ is the moment of inertia of water rotating in the plane of 
the molecule. This equation was then solved to obtain vibrational eigenstates for the water motion on the neutral and cation surfaces which, in turn, were used to compute FCFs. Within the approximation that the vibrational mode corresponding to the water motion is decoupled from the vibrational modes of the thymine, the energy associated with the water-water vibrational transitions is additive to the thymine-only spectra and the FCFs are multiplicative. Each peak appearing in the spectrum associated with the thymine moiety thus has the spectrum for the water fragment superimposed on top of it. This leads to both qualitative and quantitative changes in the theoretical spectrum, since the peaks with the largest FCFs in the water vibrational motion are the $1 \leftarrow 0$ and $2 \leftarrow 0$ peaks.

\section{Experimental details}

The experiments were performed on a molecular beam apparatus coupled to a 3 meter VUV monochromator on the Chemical Dynamics Beamline at the Advanced Light Source (ALS). The thermal vaporization source has been described recently in a publication detailing the microsolvation of DNA bases[6]. The nozzle consisted of a $0.953 \mathrm{~cm}$ diameter disk (1 mm thick) with a $100 \mu \mathrm{m}$ diameter center hole welded on to one end of a closed stainless steel tube of $0.953 \mathrm{~cm} \mathrm{OD}$ and $15.24 \mathrm{~cm}$ long. This front end of the stainless steel tube contained thymine and could be heated to between 298 and $700 \mathrm{~K}$ with a cartridge heater mounted in an aluminum heating block. The temperature of the tube was monitored with a thermocouple to the heating block. To produce the water complexes, Ar carrier gas at $58.7 \mathrm{kPa}$ was passed over a water reservoir held at room temperature and directed into the stainless steel nozzle. The temperature utilized for generating thymine vapor was $503 \mathrm{~K}$.

Shown in Figure 7 is a representative mass spectrum of microhydrated thymine recorded at a photon energy of $10 \mathrm{eV}$. The main peak is thymine followed by thymine-water clusters, where up to five waters clustered around thymine are detectable. Also observed are the thymine dimer with up to four water clusters connected to the dimer.

The clusters are ionized by tunable synchrotron radiation in the 8.0-13.0 eV region, and the ions are detected by a time-of-flight mass spectrometer. For each mass, the yield of the ions is measured as a function of photon energy, which produces PIE spectra. The typical step size for the PIE scans is $50 \mathrm{meV}$ with a dwell time of $30 \mathrm{~s}$ at a repetition rate of 10 kHz. The differentiation of the PIE curves, following the method used by Berkowitz in inter- 
preting the photoionization of methanol[48], produces a spectrum similar to a photoelectron spectrum from which information about vibrational progressions and other electronic states can be extracted. The differentiation is performed numerically after taking a five points nearest-neighbor average to reduce the effects of noise in the PIE. The accuracy of reported onset energies in the PIE spectra is $0.05 \mathrm{eV}$.

\section{RESULTS AND DISCUSSION}

\section{A. Ionization-induced geometry changes in thymine in thymine hydrates}

We begin with an overview of the structures of thymine and thymine/water clusters summarized in Figs. 2 and 3.

The minimum energy structures of the thymine monohydrates (see Fig. 1) are non-planar with the hydrogen atom of water out of the plane of thymine (the dihedral angle is $78^{0}$ for $\mathrm{T} 1)$. However, the energy difference between the planar (optimized with $\mathrm{C}_{s}$ constraint) and non-planar structures is less than $0.5 \mathrm{kcal} / \mathrm{mol}$. Moreover, the effect of non-planarity on VIE is also small (about $0.02 \mathrm{eV}$ ). Thus, we employed planar $\left(\mathrm{C}_{s}\right)$ geometries in all calculations of thymine monohydrates for computational efficiency.

The binding energy per hydrogen bond increases with addition of each water molecule. Binding energies (non ZPE-corrected, $\mathrm{D}_{e}$ ) are 11.3, 9.1, $8.9 \mathrm{kcal} / \mathrm{mol}$ for the T1, T2 and T3 monohydrates. For the dihydrates they are 23.4 and $20.1 \mathrm{kcal} / \mathrm{mol}$ for T11 and T12, and for the trihydrates (T111 and T112) they are 32.8 and $31.0 \mathrm{kcal} / \mathrm{mol}$. The optimized geometries reveal that the only structural parameters affected by hydration are those of the thymine part which is in close proximity to the water molecule (Figs. 2 and 3). The hydrogen-bonding with water results in an increase in the bond length of the $\mathrm{N}-\mathrm{H}$ and $\mathrm{C}-\mathrm{O}$ groups, and a decrease in the C-N bond length. With the addition of each water molecule the induced structural changes increase, with the largest changes in bond lengths being 0.011, 0.018 and $0.028 \AA$ for the monohydrate, dihydrate and trihydrate, respectively.

The ionization-induced structural relaxation of thymine hydrates is much larger than that of isolated thymine[9]. For example, the largest change in bond lengths of the thymine 
moiety in clusters is $0.062 \AA$, whereas for isolated thymine it is only $0.032 \AA$. A possible explanation is a large shift of water molecules towards the positively charged $\mathrm{N}-\mathrm{H}$ group and away from the negatively charged oxygen. This results in a shorter hydrogen bond between the N-H group and oxygen of water $(0.245 \AA$ for T1), and a longer hydrogen bond between the $\mathrm{C}=\mathrm{O}$ and the hydrogen of water $(0.893 \AA$ for $\mathrm{T} 1)$. As discussed below, this large structural relaxation results in vanishing FCFs for the $0 \longleftarrow 0$ transition.

\section{B. Vertical ionization energies}

The VIEs of all lowest ionized states summarized in Table I and Fig. 8 are affected by water. The first VIE in all clusters is reduced relative to thymine, and the addition of each water molecule leads to a roughly $0.1 \mathrm{eV}$ drop in VIE (except for T11-T111); however, the magnitude of the effect varies for different clusters. The changes in higher VIEs also vary for different states and cluster structures from -0.24 to $0.74 \mathrm{eV}$. For monohydrates, the largest change in the first VIE is $0.12 \mathrm{eV}$ observed in $\mathrm{T} 1$.

The first six occupied molecular orbitals of thymine monohydrates and the four MOs of diand trihydrates are localized on either thymine or water. The shapes of these MOs are almost the same as in bare thymine/water. The corresponding ionized states are of Koopmans character, e.g., the leading $R_{1}$ amplitude values are greater than 0.94 (Fig. 8). However, for the states with IEs close to water IEs, the respective MOs (e.g., the sixth orbitals of monohydrate, fifth orbital of dihydrate) become delocalized, and the corresponding wave functions become multiconfigurational (there are several $R_{1}$ amplitudes with values greater than 0.15).

\section{The origin of IE changes}

We considered several possible explanations for the observed changes of the VIE in thymine clusters: the geometry change of thymine molecule in clusters, charge transfer from thymine to water resulting in hole delocalization, and electrostatic (charge-dipole) interaction between the ionized thymine moiety and water. Below we evaluate different effects and conclude that the dominant contribution to the VIE changes is due to electrostatics. 
To estimate the effect of the change in the geometry, we computed IEs of bare thymine at the equilibrium geometry of thymine in the T1 monohydrate. The first VIE at this geometry is $9.16 \mathrm{eV}$, which is $0.03 \mathrm{eV}$ higher than that of thymine at its own equilibrium geometry $(9.13 \mathrm{eV})$. Therefore the geometry change does not explain the change of VIEs.

The degree of charge transfer between the thymine and water was evaluated using the NBO analysis. We found that the distribution of the positive charge is consistent with the shape of the corresponding Hartree-Fock orbitals. The hole is localized on thymine and the maximum charge transfer in monohydrates is only 0.027 a.u. Moreover, there is no correlation between charge transfer and the change in the IE; in fact, for T1 there is even the opposite dependence, the larger the charge transfer the smaller the change in the IE, as shown in Fig. 9.

To calculate the charge-dipole interaction energy, we employed a simple classical model following the analysis in Ref. [7]. The energy was calculated as the sum of interaction energies between the dipole of water and the partial (NBO) charges on the thymine atoms using the following equation:

$$
E=-\sum_{i} \frac{\mu \cdot q_{i}}{r_{i}^{2}} \cos \Theta
$$

where $\mu$ is the dipole moment of water, $q_{i}$ is the charge of the $i$ th thymine atom calculated by the NBO analysis, $r$ is the distance between the atom and the center of the dipole, and $\Theta$ is the angle between the dipole vector and the vector connecting the center of the dipole and the atom.

The ionization introduces a net positive charge on the thymine resulting in a new charge distribution. This leads to the change in electrostatic interaction energy, which is calculated as the difference of the interaction energy in the neutral and the ionized states. Fig. 10 shows computed IEs for different states and structures versus these charge-dipole interaction energies. There is a strong correlation between the change in the electrostatic interaction energy and the change in VIE.

We conclude that the change in VIE is explained by a simple charge-dipole interaction between the partial charges on thymine and the water dipole, which either stabilize or destabilize the ionized states relative to the neutral ground state. 


\section{Adiabatic ionization energies and FCFs}

Due to large geometry relaxations of the ionized states (see Figs. 4 and 5), the AIEs are significantly lower that VIEs. The relaxation energies for different structures are in the range of 0.4-0.6 eV, which is considerably larger than the relaxation energy of bare thymine $(0.23 \mathrm{eV})[9]$. The structures that undergo greater structural relaxation in the ionized states are characterized by a larger relaxation energy. Compare, for example, T11 and T12 (Fig. 5). The AIEs of the clusters are considerably lower than that of bare thymine and are reduced by about $0.1 \mathrm{eV}$ with addition of each water molecule.

To compare the calculated AIEs with the experimental PIE onsets, we calculated FCFs for T1 and T2, as described in Section II B. The computed intensities are convoluted by a Gaussian with width of $0.05 \mathrm{eV}$ which corresponds to the experimental width, and are shown in Fig. 11 and 12. As one can see, the relaxation in the water-thymine degrees of freedom affects the FCFs resulting in negligible intensity for the $0 \longleftarrow 0$ transitions and shifting the spectra to higher energies. The resulting PIE curves obtained by integration of FCFs are shown in Fig. 13. The computed PIE onsets are shifted by $0.03 \mathrm{eV}$ to higher energies relative to the $0 \leftarrow 0$ transitions. Moreover, the intensities at the computed onsets are so small that the apparent onsets are shifted by about $0.1 \mathrm{eV}$ to higher energies.

\section{E. Theory versus experiment}

Figs. 14 and 15 show differentiated and raw PIE spectra for $\mathrm{T}, \mathrm{T}\left(\mathrm{H}_{2} \mathrm{O}\right), \mathrm{T}\left(\mathrm{H}_{2} \mathrm{O}\right)_{2}$, $\mathrm{T}\left(\mathrm{H}_{2} \mathrm{O}\right)_{3}$ as well as the computed VIEs and AIEs for different structures. The computed IEs for different structures are summarized in Table I. For bare thymine, the experimental and computed IEs are in excellent agreement[9].

Our present experimental results from the differentiated PIE for thymine are in agreement with the previous experimental results and calculations[9]. The onset is $8.85 \pm 0.05$ $\mathrm{eV}$. The small peak at the inset arising at $8.80 \mathrm{eV}$ could come from fragmentation of higher clusters. The two peaks at $11.8 \mathrm{eV}$ and $12.2 \mathrm{eV}$ do not have theoretical counterparts (Fig. 14, T). The peak at $11.8 \mathrm{eV}$ is an experimental artifact because argon used in the gas filter to remove higher harmonics in the incident radiation has absorption lines at 11.62 
$\mathrm{eV}$ and $11.83 \mathrm{eV}$. These lines strongly perturb PIE intensities and were removed from the experimental data.

For thymine monohydrates, the experimental PIE curve onset at $8.60 \mathrm{eV}$ is in perfect agreement with the calculated AIEs for T2 $(8.65 \mathrm{eV})$ and T3 $(8.64 \mathrm{eV})$, but $0.09 \mathrm{eV}$ higher that the calculated AIE for T1 $\left(8.51 \mathrm{eV}\right.$ ) (Fig. 15, T- $\left.\mathrm{H}_{2} \mathrm{O}\right)$. This discrepancy is due to the unfavorable FCFs for T1 ionization obscuring the onset, as evidenced by the simulated PIE curves (computed by integrating Franck-Condon progressions as described in Section III D) shown in Fig. 13. We used our best estimate of adiabatic energies, i.e., including the ZPE $(-0.08 \mathrm{eV})$ and extended basis $(+0.07 \mathrm{eV})$ corrections. The calculated onsets are $8.53 \mathrm{eV}$ and $8.70 \mathrm{eV}$ for $\mathrm{T} 1$ and $\mathrm{T} 2$. They are shifted from the respective AIEs by $0.03 \mathrm{eV}$ (AIE is $8.50 \mathrm{eV}$ ) and $0.06 \mathrm{eV}$ (AIE is $8.64 \mathrm{eV}$ ). The apparent onsets are shifted even more (by $\approx 0.1 \mathrm{eV}$ ), as the intensity of the calculated PIE for T1 below $8.6 \mathrm{eV}$ is significantly smaller than at higher energies which makes it difficult to observe low energy signal in the experiment $(8.60 \mathrm{eV})$. Overall, the experimental onset at $8.60 \mathrm{eV}$ agrees very well with the computed PIE curves for T1 and T2 and the AIE of $8.56 \mathrm{eV}$ (T1). We also note that the experimental PIE rises much faster after the calculated T2 PIE onset $(8.70 \mathrm{eV})$.

The VIEs for thymine monohydrate are in good agreement with the peaks maxima in the experimental differentiated PIE curves, which correspond to experimental VIEs (Fig. 14, $\mathrm{T}-\mathrm{H}_{2} \mathrm{O}$ ). From the comparison of the experimental differentiated PIE and calculated VIEs, we conclude that all three isomers may be present in the beam.

For thymine dihydrate, the experimental onset at $8.55 \mathrm{eV}$ is in good agreement with the calculated AIE for $\mathrm{T} 11(8.52 \mathrm{eV})$ [see Fig. 15, T( $\left.\mathrm{H}_{2} \mathrm{O}\right)_{2}$ ]. The calculated AIE for the T12 structure is $8.30 \mathrm{eV}$, which is $0.25 \mathrm{eV}$ lower than the experimental onset. A possible explanation is that T12 undergoes a larger geometry relaxation upon ionization than T11. The VIEs of T11 and T12 are very close (8.89 and $8.93 \mathrm{eV}$ respectively), but T12 has a much larger relaxation energy $(0.63 \mathrm{eV}$ versus $0.37 \mathrm{eV}$ for $\mathrm{T} 11)$. This can lead to a small FCF for T12, so it is not clear if these isomers can be seen at the onset. The sharp rise of the experimental PIE curve suggests more favorable FCFs, as in T11. Finally, T12 is 3.3 $\mathrm{kcal} / \mathrm{mol}$ higher in energy than T11 and is not likely to be populated much in the molecular 
beam.

The theoretical VIEs for both thymine dihydrate structures agree well with the experimental differentiated PIE peaks [Fig. 14, $\mathrm{T}\left(\mathrm{H}_{2} \mathrm{O}\right)_{2}$ ]. There are a few experimental peaks that do not have theoretical VIE counterparts suggesting that more than the two $\mathrm{T}\left(\mathrm{H}_{2} \mathrm{O}\right)_{2}$ isomers considered here present in the beam.

The theoretical AIE for thymine trihydrate T111 is $8.35 \mathrm{eV}$, which is close to the experimental onset at $8.40 \mathrm{eV}$ [(Fig. 15, $\left.\mathrm{T}\left(\mathrm{H}_{2} \mathrm{O}\right)_{3}\right]$. The theoretical AIE for the T112 structure is $8.21 \mathrm{eV}$, which is $0.19 \mathrm{eV}$ lower than the experimental value. Possible explanations of the difference are unfavorable FCFs and smaller concentration of T112 in the molecular beam relative to T111. The theoretical VIEs for both trihydrates are in good agreement with the experimental PIE curve, which can be seen in Fig. 14, $\mathrm{T}\left(\mathrm{H}_{2} \mathrm{O}\right)_{3}$. As suggested for $\mathrm{T}\left(\mathrm{H}_{2} \mathrm{O}\right)$ and $\mathrm{T}\left(\mathrm{H}_{2} \mathrm{O}\right)_{2}$, there could also be minor contributions from other structures which are not considered here.

\section{CONCLUSIONS}

We have presented a combined theoretical and experimental study of the ionized clusters of thymine with one, two and three water molecules. The PIE onsets are $8.85 \pm 0.05$ $\mathrm{eV}, 8.60 \pm 0.05 \mathrm{eV}, 8.55 \pm 0.05 \mathrm{eV}, 8.40 \pm 0.05 \mathrm{eV}$ for bare thymine, thymine monohydrate, dihydrate and trihydrate, respectively. The computed EOM-IP-CCSD AIEs are $8.90 \mathrm{eV}$, 8.51-8.64 eV, 8.30-8.52 eV and 8.21-8.35 eV for bare thymine, thymine monohydrates, dihydrates and trihydrates, respectively.

All thymine microhydrates undergo significant structural relaxation upon ionization, which results in higher relaxation energies for microhydrates than for the bare thymine molecule, e.g., the computed relaxation energies for thymine, $\mathrm{T}-\mathrm{H}_{2} \mathrm{O}, \mathrm{T}\left(\mathrm{H}_{2} \mathrm{O}\right)_{2}$ and $\mathrm{T}\left(\mathrm{H}_{2} \mathrm{O}\right)_{3}$ are $0.23,0.40-0.50,0.37-0.63,0.53-0.62 \mathrm{eV}$, respectively.

We found that due to the large structural relaxation in hydrated species, the FCFs for the $0 \leftarrow 0$ transitions are small thus shifting the apparent onsets to higher energy $(0.03-0.10 \mathrm{eV})$. The agreement between theoretical and measured PIEs is good, however, due to unfavorable FCFs the PIE onsets overestimate the AIEs. The theoretical VIEs are in good agreement with the maxima of the differentiated PIE curves.

There is a significant effect of microhydration on the AIE of thymine. Addition of the first 
water molecule decreases the AIE as much as $0.39 \mathrm{eV}$, the second water molecule decreases the AIE as much as $0.35 \mathrm{eV}$, and the third one - as much as $0.31 \mathrm{eV}$. VIEs also are affected by the addition of water. The effect is different for different structures and ionized states. The change of VIE depends on the shape of the corresponding MOs and the position of the water and is dominated by electrostatic (charge-dipole) interactions. We found that there is no significant charge transfer from thymine to water, and that the changes of VIEs due to hydration-induced structural changes in the thymine moiety are small.

\section{Acknowledgments}

This work was conducted under the auspices of the iOpenShell Center for Computational Studies of Electronic Structure and Spectroscopy of Open-Shell and Electronically Excited Species (iopenshell.usc.edu) supported by the National Science Foundation through the CRIF:CRF CHE-0625419+0624602+0625237 and CHE-0951634 (A.I.K.) grant. O.K. and M.A. acknowledge support by the Director, Office of Energy Research, Office of Basic Energy Sciences, Chemical Sciences Division of the U.S. Department of Energy under contract No. DE-AC02-05CH11231.

[1] Cauët, E. ; Valiev, M. ; Weare, J.H. J. Phys. Chem. B 2010, 114, 5886.

[2] Slavíček, P. ; Winter, B. ; Faubel, M. ; Bradforth, S.E. ; Jungwirth, P. J. Am. Chem. Soc. 2009, 131, 6460 .

[3] Golubeva, A.A. ; Krylov, A.I. Phys. Chem. Chem. Phys. 2009, 11, 1303.

[4] Zadorozhnaya, A.A. ; Krylov, A.I. J. Chem. Theory Comput. 2010, 6, 705.

[5] Zadorozhnaya, A.A. ; Krylov, A.I. J. Phys. Chem. A 2010, 114, 2001.

[6] Belau, L. ; Wilson, K.R. ; Leone, S.R. ; Ahmed, M. J. Phys. Chem. A 2007, 111, 7562.

[7] Bravaya, K.B. ; Kostko, O. ; Ahmed, M. ; Krylov, A.I. Phys. Chem. Chem. Phys. 2010, 12, 2292.

[8] Kostko, O. ; Bravaya, K.B. ; Krylov, A.I. ; Ahmed, M. Phys. Chem. Chem. Phys. 2010, 12, 2860.

[9] Bravaya, K.B. ; Kostko, O. ; Dolgikh, S. ; Landau, A. ; Ahmed, M. ; Krylov, A.I. J. Phys. 
Chem. A 2010, 114, 12305.

[10] Kim, S.K. ; Lee, W. ; Herschbach, D.R. J. Phys. Chem. 1996, 100, 7933.

[11] Choi, K.-W. ; Lee, J.-H. ; Kim, S.K. J. Am. Chem. Soc. 2005, 127, 15674.

[12] Close, D.M. ; Crespo-Hernández, C.E. ; Gorb, L. ; Leszczynski, J. J. Phys. Chem. A 2006, 110,7485 .

[13] Close, D.M. ; Crespo-Hernández, C.E. ; Gorb, L. ; Leszczynski, J. J. Phys. Chem. Lett. 2008, 112, 4405 .

[14] Smith, V.R. ; Samoylova, E. ; Ritze, H.H. ; Radloff, W. ; Schultz, T. Phys. Chem. Chem. Phys. 2010, 12, 9632.

[15] Sobolewski, A.L. ; Domcke, W. Phys. Chem. Chem. Phys. 2007, 9, 3818.

[16] Sobolewski, A. L. ; Domcke, W. ; Dedonder-Lardeuxc, C. ; Jouvet, C. Phys. Chem. Chem. Phys. 2002, 4, 1093.

[17] Sobolewski, A.L. ; Domcke, W. J. Phys. Chem. A 2001, 105, 9275.

[18] Ritze, H.-H. ; Lippert, H. ; Samoylova, E. ; Smith, V.R. ; Hertel, I.V. ; Radloff, W. ; Schultz, T. J. Chem. Phys. 2005, 122, 224320.

[19] He, Y. ; Wu, C. ; Kong, W. J. Phys. Chem. A 2004, 108, 943.

[20] Shukla, M.K. ; Leszczynski, J. J. Phys. Chem. A 2002, 106, 11338.

[21] Yoshikawa, A. ; Matsika, S. Chem. Phys. 2008, 347, 393.

[22] Marian, C.M. ; Schneider, F. ; Kleinschmidt, M. ; Tatchen, J. Eur. Phys. J. D 2002, 20, 357.

[23] Pieniazek, P.A. ; Arnstein, S.A. ; Bradforth, S.E. ; Krylov, A.I. ; Sherrill, C.D. J. Chem. Phys. 2007, 127, 164110.

[24] Krylov, A.I. Annu. Rev. Phys. Chem. 2008, 59, 433.

[25] Chai, J.-D. ; Head-Gordon, M. J. Chem. Phys. 2008, 128, 084106.

[26] Chai, J.-D. ; Head-Gordon, M. Phys. Chem. Chem. Phys. 2008, 10, 6615.

[27] Sinha, D. ; Mukhopadhyay, D. ; Mukherjee, D. Chem. Phys. Lett. 1986, 129, 369.

[28] Pal, S. ; Rittby, M. ; Bartlett, R.J. ; Sinha, D. ; Mukherjee, D. Chem. Phys. Lett. 1987, 137, 273.

[29] Stanton, J.F. ; Gauss, J. J. Chem. Phys. 1994, 101, 8938.

[30] Nooijen, M. ; Bartlett, R.J. J. Chem. Phys. 1995, 102, 3629.

[31] Pieniazek, P.A. ; Bradforth, S.E. ; Krylov, A.I. J. Chem. Phys. 2008, 129, 074104.

[32] Oana, C.M. ; Krylov, A.I. J. Chem. Phys. 2007, 127, 234106. 
[33] Pieniazek, P.A. ; Krylov, A.I. ; Bradforth, S.E. J. Chem. Phys. 2007, $127,044317$.

[34] Pieniazek, P.A. ; VandeVondele, J. ; Jungwirth, P. ; Krylov, A.I. ; Bradforth, S.E. J. Phys. Chem. A 2008, 112, 6159 .

[35] Pieniazek, P.A. ; Sundstrom, E.J. ; Bradforth, S.E. ; Krylov, A.I. J. Phys. Chem. A 2009, $113,4423$.

[36] Kamarchik, E. ; Kostko, O. ; Bowman, J.M. ; Ahmed, M. ; Krylov, A.I. J. Chem. Phys. 2010, 132, 194311.

[37] Larsen, H. ; Hald, K. ; Olsen, J. ; Jørgensen, P. J. Chem. Phys. 2001, 115, 3015.

[38] Manohar, P.U. ; Stanton, J.F. ; Krylov, A.I. J. Chem. Phys. 2009, 131, 114112.

[39] Rejnek, J. ; Hanus, M. ; Kabeláç, M. ; Ryjék, F. ; Hobza, P. Phys. Chem. Chem. Phys. 2005, 7, 2006.

[40] Weigend, F. ; Häser, M. ; Patzelt, H. ; Ahlrichs, R. Chem. Phys. Lett. 1998, $294,143$.

[41] Dunning, T.H. J. Chem. Phys. 1989, 90, 1007.

[42] Krishnan, R. ; Binkley, J.S. ; Seeger, R. ; Pople, J.A. J. Chem. Phys. 1980, 72, 650.

[43] Landau, A. ; Khistyaev, K. ; Dolgikh, S. ; Krylov, A.I. J. Chem. Phys. 2010, 132, 014109.

[44] NBO 5.0. Glendening, E.D. ; Badenhoop, J.K. ; Reed, A.E. ; Carpenter, J.E. ; Bohmann, J.A. ; Morales, C.M. ; Weinhold, F. Theoretical Chemistry Institute, University of Wisconsin, Madison, WI, 2001.

[45] Y. Shao, L.F. Molnar, Y. Jung, J. Kussmann, C. Ochsenfeld, S. Brown, A.T.B. Gilbert, L.V. Slipchenko, S.V. Levchenko, D.P. O’Neil, R.A. Distasio Jr, R.C. Lochan, T. Wang, G.J.O. Beran, N.A. Besley, J.M. Herbert, C.Y. Lin, T. Van Voorhis, S.H. Chien, A. Sodt, R.P. Steele, V.A. Rassolov, P. Maslen, P.P. Korambath, R.D. Adamson, B. Austin, J. Baker, E.F.C. Bird, H. Daschel, R.J. Doerksen, A. Drew, B.D. Dunietz, A.D. Dutoi, T.R. Furlani, S.R. Gwaltney, A. Heyden, S. Hirata, C.-P. Hsu, G.S. Kedziora, R.Z. Khalliulin, P. Klunziger, A.M. Lee, W.Z. Liang, I. Lotan, N. Nair, B. Peters, E.I. Proynov, P.A. Pieniazek, Y.M. Rhee, J. Ritchie, E. Rosta, C.D. Sherrill, A.C. Simmonett, J.E. Subotnik, H.L. Woodcock III, W. Zhang, A.T. Bell, A.K. Chakraborty, D.M. Chipman, F.J. Keil, A. Warshel, W.J. Herhe, H.F. Schaefer III, J. Kong, A.I. Krylov, P.M.W. Gill, M. Head-Gordon Phys. Chem. Chem. Phys. 2006, 8,3172 .

[46] Berger, R. ; Fischer, C. ; Klessinger, M. J. Phys. Chem. A 1998, 102, 7157.

[47] Mozhayskiy, V.A. ; Krylov, A.I. ; 
ezSpectrum, http://iopenshell.usc.edu/downloads/.

[48] Berkowitz, J. J. Chem. Phys. 1978, 69, 3044. 
TABLE I: Vertical and adiabatic IEs (eV) of thymine and thymine-water clusters computed by EOM-IP-CCSD/cc-pVTZ.

\begin{tabular}{|c|c|c|c|c|c|c|c|c|}
\hline \multirow[t]{2}{*}{ State } & \multirow[t]{2}{*}{$\mathrm{T}$} & \multicolumn{3}{|c|}{$\mathrm{T}-\mathrm{H}_{2} \mathrm{O}$} & \multicolumn{2}{|c|}{$\mathrm{T}\left(\mathrm{H}_{2} \mathrm{O}\right)_{2}$} & \multicolumn{2}{|c|}{$\mathrm{T}\left(\mathrm{H}_{2} \mathrm{O}\right)_{3}$} \\
\hline & & $\mathrm{T} 1$ & $\mathrm{~T} 2$ & T3 & $\mathrm{T} 11$ & $\mathrm{~T} 12$ & $\mathrm{~T} 111$ & $\mathrm{~T} 112$ \\
\hline 1 & 9.13 & $9.01(0.12)$ & $9.05(0.08)$ & $9.08(0.05)$ & $8.89(0.24)$ & $8.93(0.20)$ & $8.88(0.25)$ & $8.83(0.30)$ \\
\hline 2 & 10.13 & $10.10(0.03)$ & $10.17(-0.04)$ & $9.97(0.16)$ & $10.10(0.03)$ & $10.15(-0.02)$ & $10.03(0.10)$ & $10.08(0.05)$ \\
\hline 3 & 10.52 & $10.51(0.01)$ & $10.36(0.16)$ & $10.34(0.18)$ & $10.44(0.08)$ & $10.36(0.16)$ & $10.48(0.04)$ & $10.30(0.22)$ \\
\hline 4 & 11.04 & $11.1(-0.06)$ & $10.87(0.17)$ & $11.03(0.01)$ & $11.04(0.00)$ & $10.92(0.12)$ & $11.08(-0.04)$ & $10.88(0.14)$ \\
\hline $5\left(\mathrm{H}_{2} \mathrm{O}\right)$ & 12.39 & $12.30(0.09)$ & $11.94(0.45)$ & $11.97(0.42)$ & $11.93(0.74)$ & $11.93(0.74)$ & 11.97 & 11.85 \\
\hline 6 & 12.67 & $12.53(0.14)$ & $12.58(-0.19)$ & $12.63(-0.24)$ & $12.38(0.01)$ & $12.21(0.18)$ & 12.18 & 11.87 \\
\hline 7 & 13.82 & $13.70(0.12)$ & $13.60(0.22)$ & $13.55(0.27)$ & $13.50(0.32)$ & $13.52(0.60)$ & 12.31 & 12.31 \\
\hline 1st AIE & 8.90 & $8.51(0.39)$ & $8.65(0.25)$ & $8.64(0.26)$ & $8.52(0.38)$ & $8.30(0.60)$ & $8.35(0.55)$ & $8.21(0.79)$ \\
\hline$\Delta E^{a}$ & 0.23 & 0.50 & 0.40 & 0.44 & 0.37 & 0.63 & 0.53 & 0.62 \\
\hline
\end{tabular}




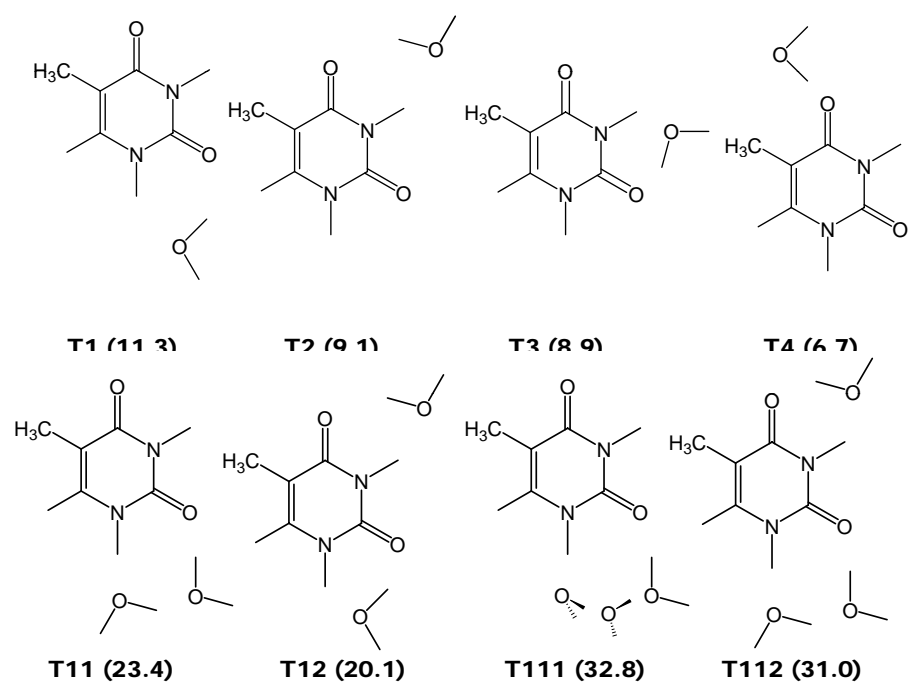

FIG. 1: Structures and binding energies $\left(\mathrm{D}_{e}, \mathrm{kcal} / \mathrm{mol}\right)$ of the considered thymine-water monohydrates, dihydrates and trihydrates, CCSD/cc-pVTZ at RI-MP2/cc-pVTZ geometry.
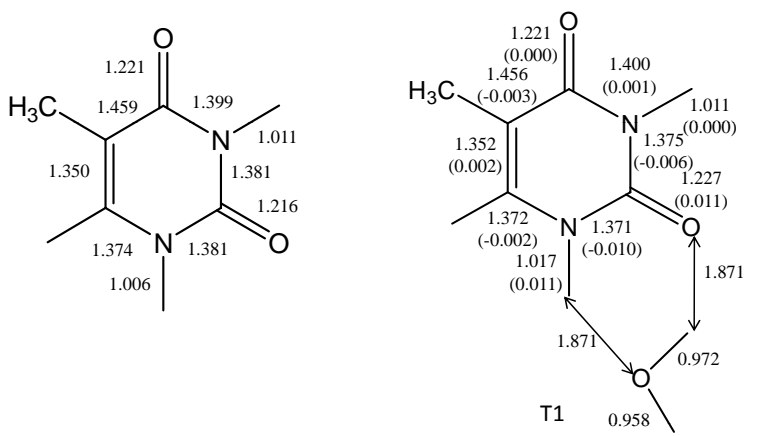

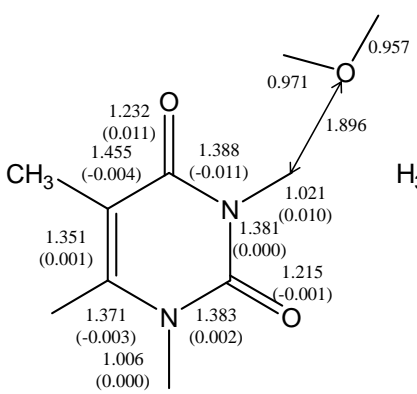

$\mathrm{T} 2$

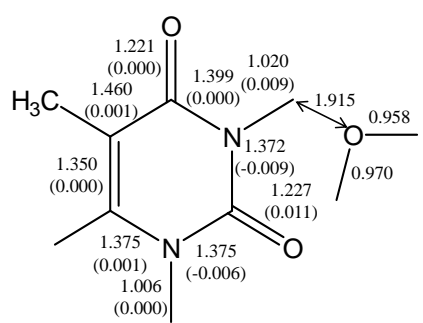

T3

FIG. 2: Equilibrium structures of thymine and three thymine monohydrates optimized by RIMP2/cc-pVTZ. Bond lengths and changes in bond lengths due to interactions with water are shown (in $\AA$ ). 


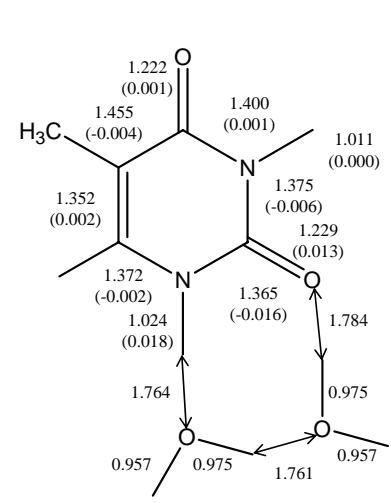

$\mathrm{T} 11$

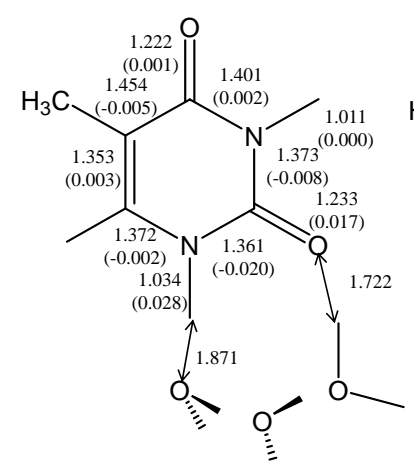

$\mathrm{T} 111$

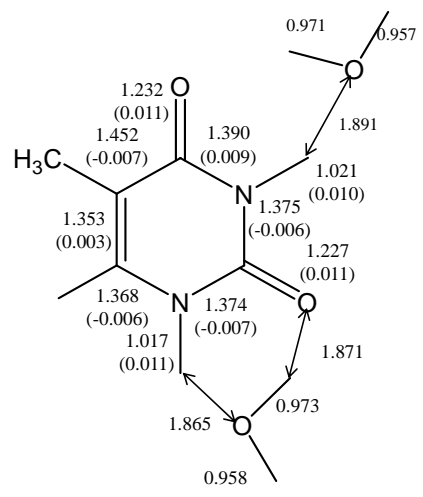

$\mathrm{T} 12$

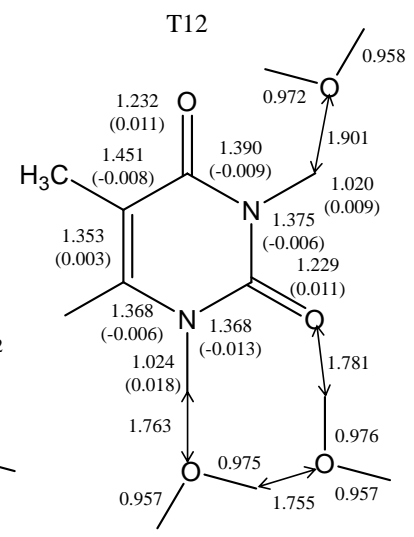

$\mathrm{T} 112$

FIG. 3: Equilibrium structures of thymine dihydrate and trihydrate optimized by RI-MP2/ccpVTZ. Bond lengths and changes in bond lengths due to microhydration are shown (in $\AA$ ).

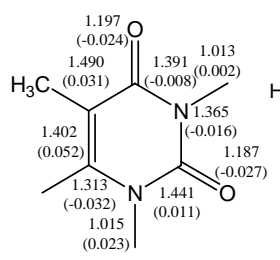

$\mathrm{T}$

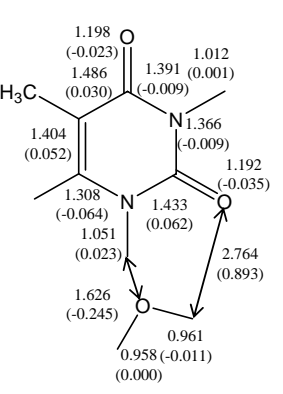

$\mathrm{T} 1$

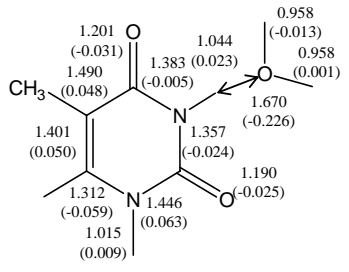

$\mathrm{T} 2$ and $\mathrm{T} 3$

FIG. 4: Equilibrium structures of ionized thymine and thymine monohydrates optimized by $\omega$ B97XD/cc-pVTZ. Bond lengths and changes in bond lengths due to ionization are shown (in $\AA$ ). 

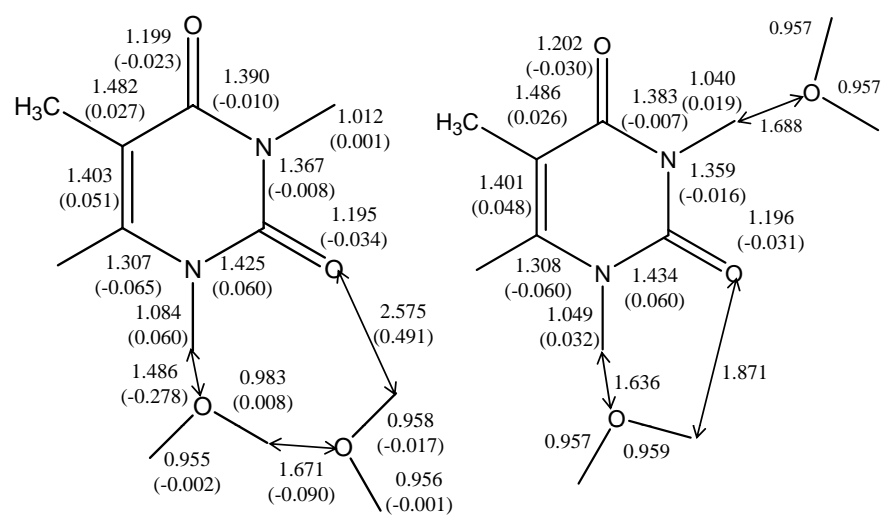

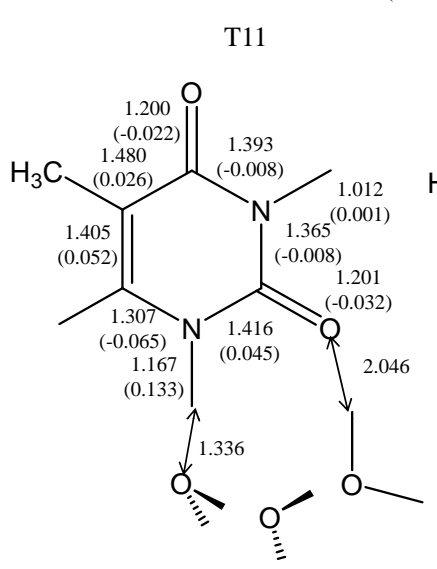

T111

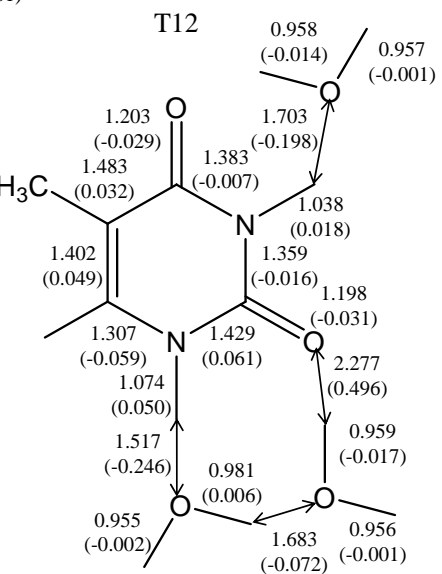

T112

FIG. 5: Equilibrium structures of ionized thymine and thymine di- and trihydrates optimized by $\omega$ B97X-D/cc-pVTZ. Bond lengths and changes in bond lengths due to ionization are shown (in $\AA$ ). 


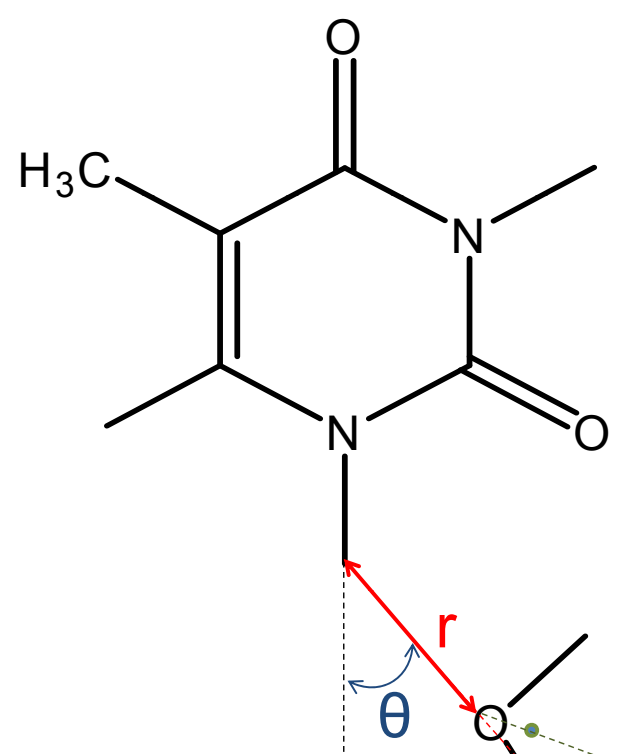

FIG. 6: Coordinates describing relative water-thymine mption.

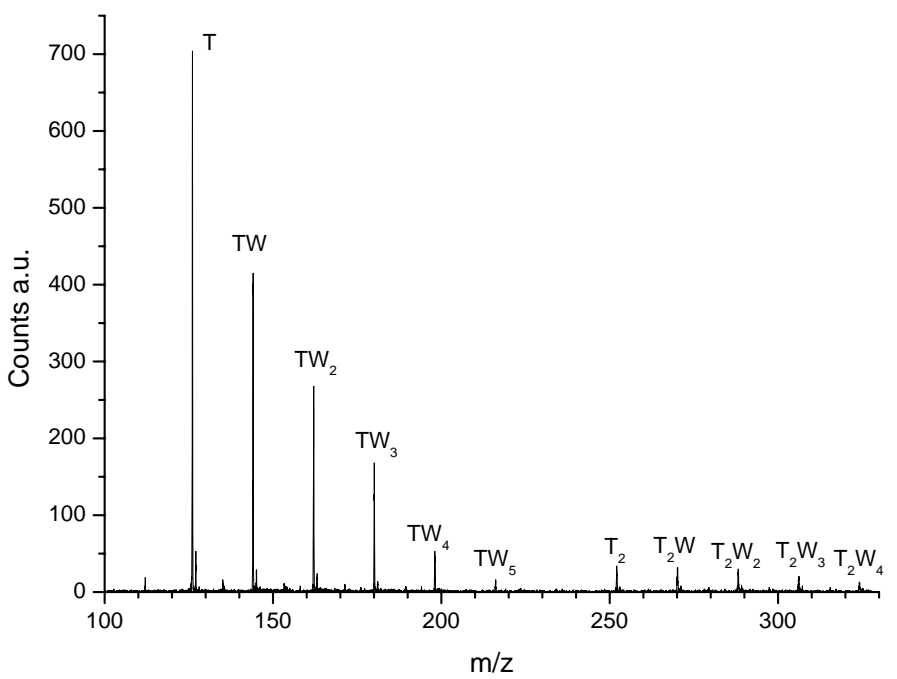

FIG. 7: Mass spectrum of microhydrated thymine recorded at a photon energy of $10 \mathrm{eV}$. 


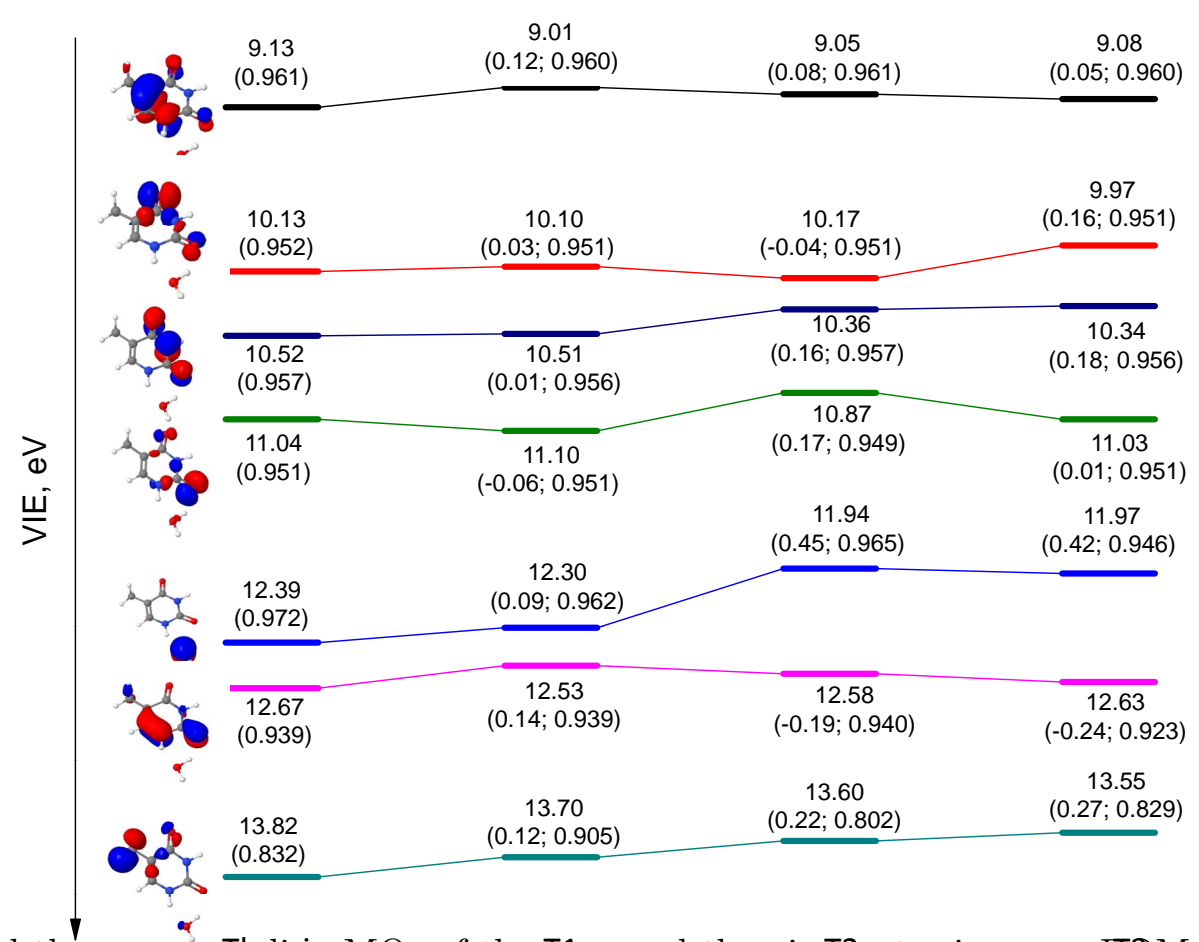

FIG. 8: VIEs (eV) and the correspoh⿲linigeMOs of thymine and thymind-2water isomers, EB)M-IPCCSD/cc-pVTZ. Changes in VIEs due to microhydration and the leading EOM-IP amplitude are given in parentheses.

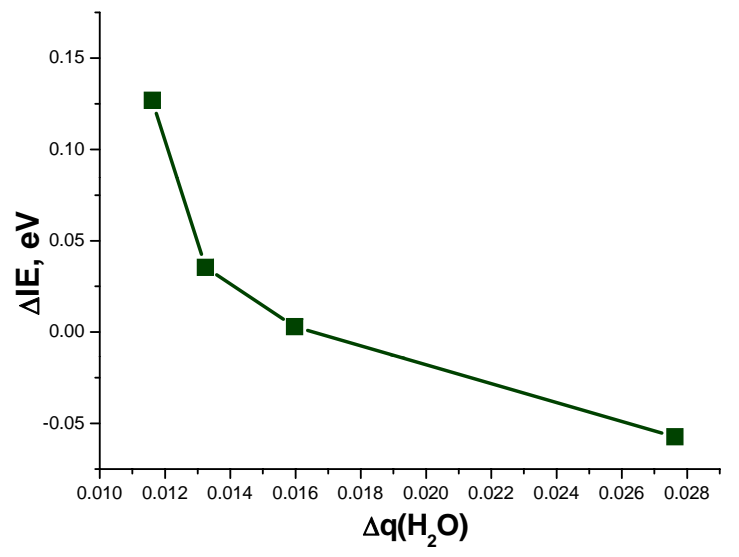

FIG. 9: Changes in VIEs versus the degree of charge transfer to water in different ionized states of T1. 


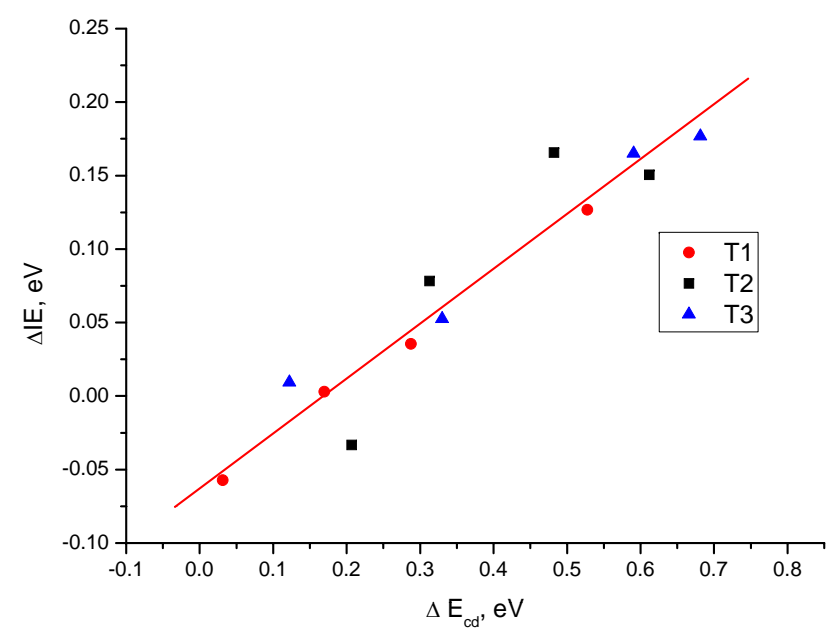

FIG. 10: Changes in VIEs versus charge-dipole interaction energy between the charges on thymine and the dipole moment of water molecule.

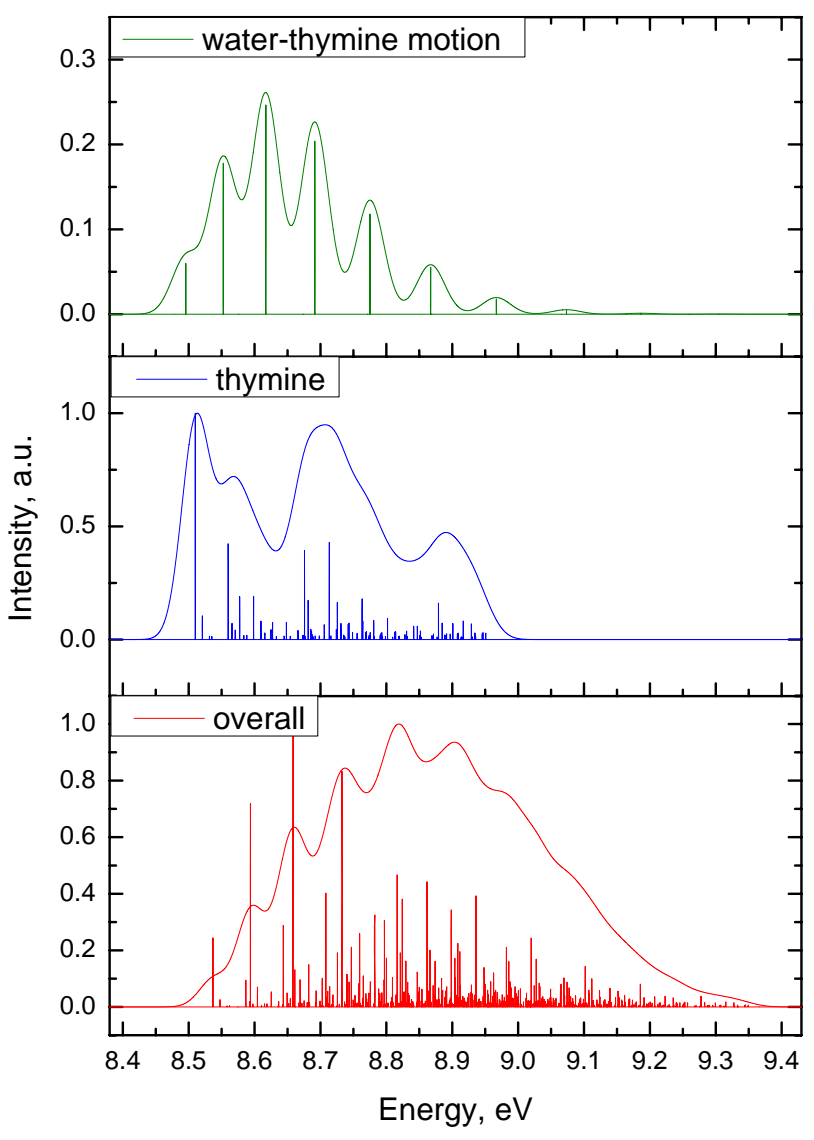

FIG. 11: Calculated FCFs for the first ionized state of T1 (lower panel). Upper panel: FCFs due to water-thymine motion (undefined scale); middle panel: FCFs due to thymine moiety. 


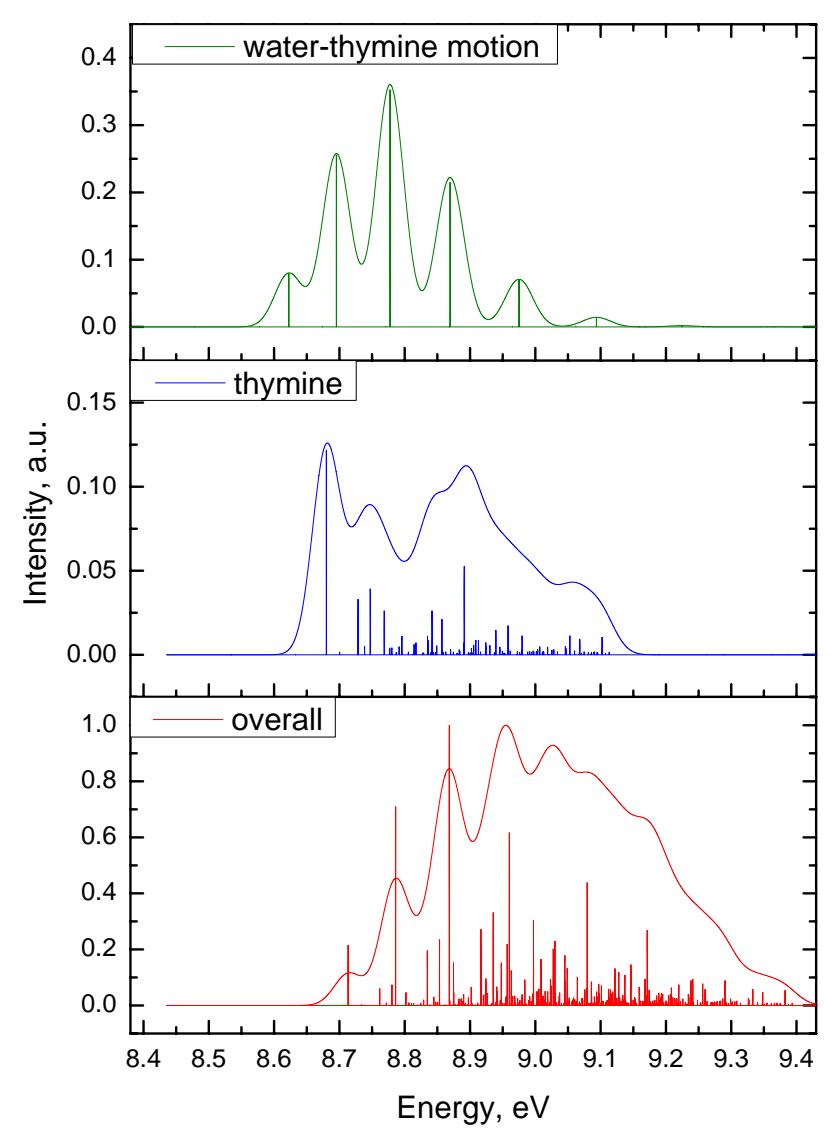

FIG. 12: Calculated FCFs factors for the first ionized state of T2 (lower panel). Upper panel: FCFs due to water-thymine motion (undefined scale); middle panel: FCFs due to thymine moiety. 


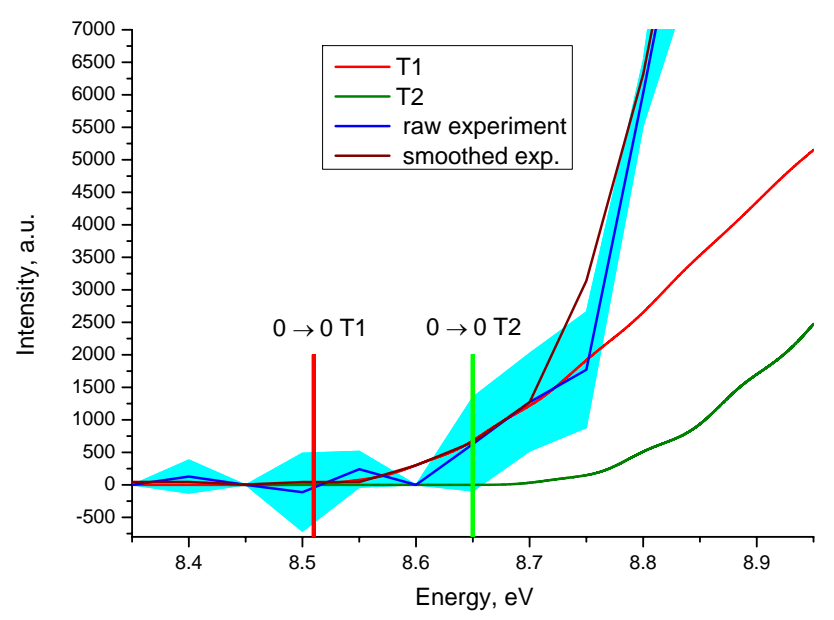

FIG. 13: The experimental (raw and smoothed data, and error bars) and calculated (T1 and T2) PIE curves. The respective adiabatic $0 \leftarrow 0$ transitions are also shown.
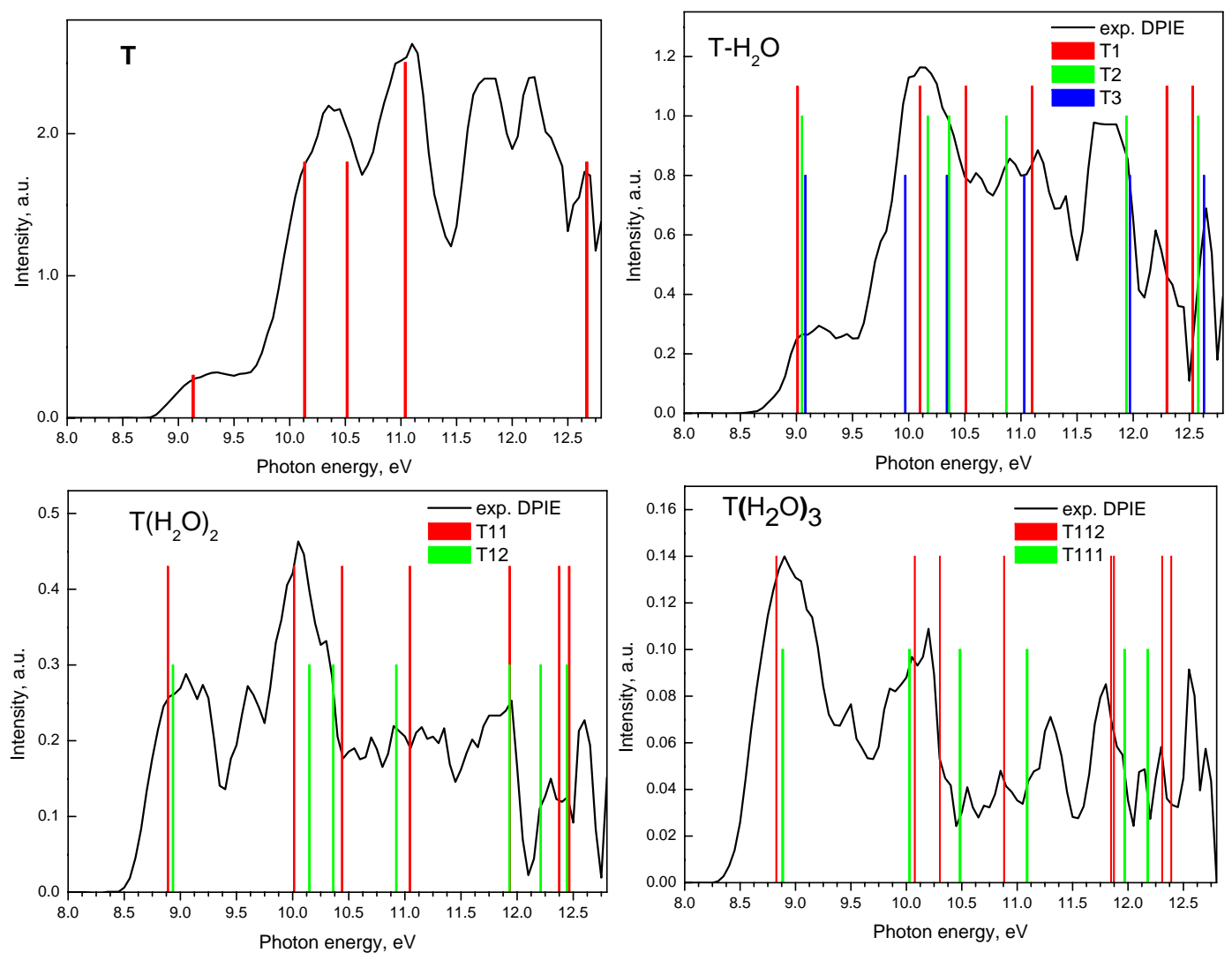

FIG. 14: The differentiated PIE (DPIE) curves and calculated VIEs for thymine and thymine clusters with one, two, and three water molecules. 

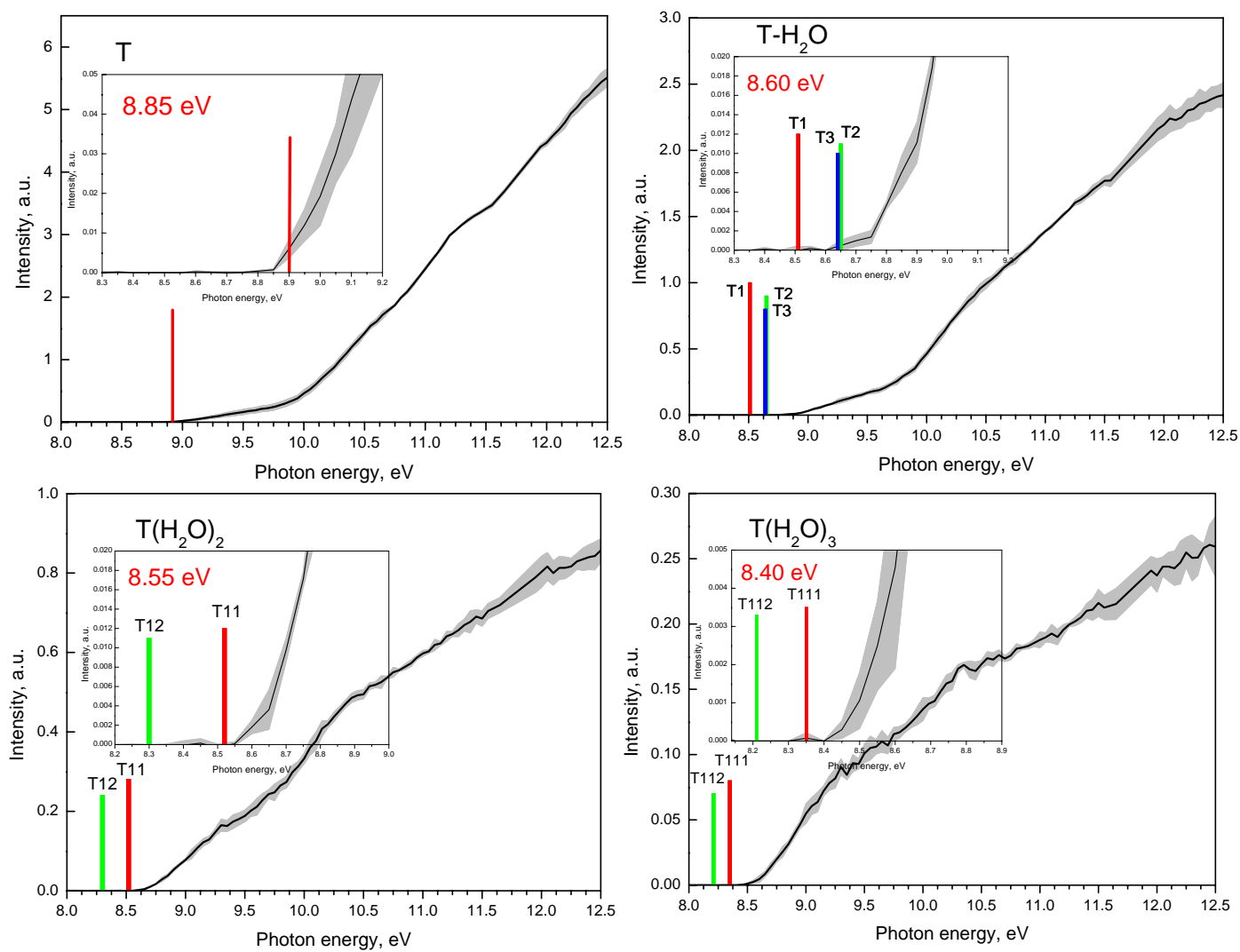

FIG. 15: The PIE curves and calculated AIEs for thymine and thymine clusters with one, two, and three water molecules. 